\title{
Preoperative Thrombocytopenia May Predict Poor Surgical Outcome after Extended Hepatectomy
}

\author{
Mohammad Golriz $\left(\mathbb{D}\right.$, ${ }^{1,2}$ Omid Ghamarnejad $\left(\mathbb{D}\right.$, ${ }^{1}$ Elias Khajeh $(\mathbb{D}){ }^{1}$ \\ Mohammadsadegh Sabagh, ${ }^{1}$ Markus Mieth, ${ }^{1}$ Katrin Hoffmann, ${ }^{1,2}$ Alexis Ulrich, ${ }^{1}$ \\ Thilo Hackert, ${ }^{1}$ Karl Heinz Weiss, ${ }^{2,3}$ Peter Schirmacher, ${ }^{2,4}$ \\ Markus W. Büchler, ${ }^{1}$ and Arianeb Mehrabi ${ }^{1,2}$ \\ ${ }^{1}$ Department of General, Visceral, and Transplantation Surgery, University of Heidelberg, Heidelberg, Germany \\ ${ }^{2}$ Liver Cancer Center Heidelberg (LCCH), Heidelberg, Germany \\ ${ }^{3}$ Department of Gastroenterology and Hepatology, University of Heidelberg, Heidelberg, Germany \\ ${ }^{4}$ Institute of Pathology, University of Heidelberg, Heidelberg, Germany \\ Correspondence should be addressed to Arianeb Mehrabi; arianeb.mehrabi@med.uni-heidelberg.de
}

Received 6 April 2018; Revised 12 June 2018; Accepted 27 June 2018; Published 1 November 2018

Academic Editor: Tatsuo Kanda

Copyright ( 2018 Mohammad Golriz et al. This is an open access article distributed under the Creative Commons Attribution License, which permits unrestricted use, distribution, and reproduction in any medium, provided the original work is properly cited.

\begin{abstract}
Background. It is a novel idea that platelet counts may be associated with postoperative outcome following liver surgery. This may help in planning an extended hepatectomy $(\mathrm{EH})$, which is a surgical procedure with high morbidity and mortality. Aim. The aim of this study was to evaluate the predictive potential of platelet counts on the outcome of EH in patients without portal hypertension, splenomegaly, or cirrhosis. Methods. A series of 213 consecutive patients underwent EH (resection of $\geq$ five liver segments) between 2001 and 2016. The association of preoperative platelet counts with posthepatectomy liver failure (PHLF), morbidity (based on Clavien-Dindo classification), and 30-day mortality was evaluated using multivariate analysis. Results. PHLF was detected in $26.3 \%$ of patients, major complications in $26.8 \%$, and 30 -day mortality in $11.3 \%$ of patients. Multivariate analysis revealed that the preoperative platelet count is an independent predictor of PHLF (odds ratio [OR] 4.4, 95\% confidence interval [CI] 1.3-15.0, $p=0.020$ ) and 30-day mortality (OR 4.4, 95\% CI 1.1-18.8, $p=0.043$ ). Conclusions. Preoperative platelet count is associated with PHLF and mortality following extended liver resection. This association was independent of other related parameters. Prospective studies are needed to evaluate the predictive role and to determine the impact of preoperative correction of platelet count on postoperative outcomes after EH.
\end{abstract}

\section{Introduction}

Extended hepatectomy (EH) is the only curative treatment for large primary or bilobar metastatic hepatic malignancies that improves long-term survival $[1,2]$. Surgical developments, better patient selection, and improvements in perioperative care have increased the number of $\mathrm{EH}$ procedures being performed $[3,4]$. However, the rate of postoperative morbidity is high following $\mathrm{EH}$, especially posthepatectomy liver failure (PHLF) [5, 6]. Preoperative predictive factors of PHLF may play an important role in assessing the risk of post-EH morbidity and mortality.
Several studies have evaluated different predictive factors for PHLF and other postoperative clinical outcomes [7-12]. Recently, the association of the perioperative (preoperative or immediate postoperative) platelet count with PHLF and postoperative mortality has been investigated [13-16]. However, findings have been controversial; some studies have shown a negative association between perioperative platelet counts and postoperative morbidity and mortality, while findings from other studies have indicated no association [13, 15, 17]. Although low platelet count is related to intraoperative poor outcome such as bleeding, it may have a direct impact on posthepatectomy outcomes by promoting liver regeneration 
and lowering the risk of PHLF. To the best of our knowledge, the association between preoperative platelet count and postoperative outcome has not been investigated exclusively in $\mathrm{EH}$, which has a higher postoperative morbidity and mortality compared with minor hepatectomy.

The aim of this study was to investigate the association of the preoperative platelet count and postoperative clinical outcomes following $\mathrm{EH}$ in patients without portal hypertension, splenomegaly, or cirrhosis. To do this, we investigated the effect of preoperative thrombocytopenia on PHLF, morbidity, and mortality after EH.

\section{Patients and Methods}

2.1. Study Population. We investigated all patients who underwent liver resection to treat primary, metastatic, or benign liver disease at the department of General, Visceral, and Transplantation Surgery at the University of Heidelberg between October 2001 and September 2016. All patients were followed up until September 2017. Only patients who underwent $\mathrm{EH}$ were included in the study. $\mathrm{EH}$ was defined as resection of five or more hepatic segments based on the Brisbane 2000 classification [18]. Patients under 18 years old and patients who underwent a two-stage hepatectomy (portal vein embolization or associated liver partition and portal vein ligation for staged hepatectomy) were excluded. At the end, a total of 213 patients were included in our study. Furthermore, preoperative imaging reports, intraoperative flowmetry, and postoperative histopathological examinations were screened to assess splenomegaly, portal hypertension, and cirrhosis, respectively. Demographic and baseline clinical characteristics, as well as data on the surgical procedure and perioperative course, were prospectively collected and analyzed. This study was approved by the independent ethics committee of the University of Heidelberg. All procedures were conducted in accordance with the most recent revision of the Declaration of Helsinki.

\subsection{Definition and Classification of Postoperative Outcomes.} PHLF was diagnosed and graded (grade A, B, or C) according to the proposed definition by the International Study Group of Liver Surgery (ISGLS) [19]. Briefly, PHLF was defined as an increased international normalized ratio (INR), the need for coagulation factors to maintain normal INR, and hyperbilirubinemia on or after postoperative day 5. Hyperbilirubinemia was defined as a serum bilirubin concentration greater than $1 \mathrm{mg} / \mathrm{dl}$ and increased INR was defined as an INR greater than 1.2. In patients with preoperative hyperbilirubinemia or increased INR, PHLF was defined as an increase in serum bilirubin levels or INR on or after postoperative day 5 .

The severity of postoperative morbidities were classified as grade I to V based on the Clavien-Dindo classification [20]. Grade I and II morbidities were defined as minor and grade III and IV morbidities were defined as major. Postoperative mortality was defined as all-cause death occurring within the first 30 days after surgery.

2.3. Preoperative Evaluations. All preoperative clinical evaluations including medical history, physical examination, and laboratory findings were recorded. All patients underwent cross-sectional contrast-enhanced computed tomography or magnetic resonance imaging of the chest, abdomen, and pelvis to assess the resectability of the tumor and to plan the hepatectomy. The preoperative platelet count was measured on the day of surgery and thrombocytopenia was defined as a platelet count $<150 \times 10^{9} / \mathrm{L}$.

2.4. Statistical Analysis. Statistical analysis was performed using IBM SPSS Statistics for Windows, Version 22.0 (IBM Corp. Released 2013. Armonk, NY). Categorical data were presented as frequencies and proportions, and continuous data were presented as means \pm standard deviations. Categorical data were compared using chi-square test of association or Fisher's exact test. Continuous data were compared using Student's t-test. Univariate and multivariate logistic regression analyses were performed to determine independent preoperative predictive factors of PHLF, major morbidity, and 30 -day mortality. Variables with a $p$ value $<0.1$ from the univariate analysis were included in the multivariate regression analysis. Results of univariate and multivariate analyses were reported as odds ratio (OR) with $95 \%$ confidence interval (CI). If thrombocytopenia was confirmed in the multivariate analysis, a comparison between patients with platelet counts $<150 \times 10^{9} / \mathrm{L}$ and platelet counts $\geq 150 \times 10^{9} / \mathrm{L}$ was performed. One-year and three-year patient survival were analyzed using the Kaplan-Meier method. Patients who were lost to follow up were censored. The mean patient survival in the two groups was compared using the log-rank test. A two-sided $p$ value less than 0.05 was considered significant in all analyses.

\section{Results}

The mean age of patients was $60.8 \pm 11.7$ years and $50.7 \%$ were female. Primary hepatic malignancy was the most common indication for $\mathrm{EH}$ (57.7\% of patients), and $35.8 \%$ of patients received preoperative systemic chemotherapy. PHLF was detected in $26.3 \%$ of patients, major complications (grade III-IV) in $26.8 \%$ of patients, and 30 -day mortality in $11.3 \%$ of patients. Detailed patient demographics and clinical data are shown in Table 1.

Seventeen patients $(8.0 \%)$ had a preoperative platelet count of $<150 \times 10^{9} / \mathrm{L}$ (mean platelet count $=122.3 \pm 22.3 \times$ $10^{9}$ per $\left.\mathrm{L}\right)$, and the remaining $196(92.0 \%)$ patients had a preoperative platelet count of $\geq 150 \times 10^{9} / \mathrm{L}$ (mean platelet count $=315.5 \pm 114.0 \times 10^{9}$ per L). Baseline characteristics and clinical outcome of the patients with preoperative thrombocytopenia are shown in Table 2. Nine of 17 patients $(52.9 \%)$ with preoperative platelet count $<150 \times 10^{9} / \mathrm{L}$ were diagnosed with primary liver malignancy (cholangiocarcinoma). Furthermore, preoperative imaging, intraoperative flowmetry, and postoperative histopathological examinations revealed no splenomegaly, portal hypertension, or cirrhosis in the thrombocytopenia group. There were only seven patients with Child-Pugh score A cirrhosis in platelet count $\geq 150$ $\mathrm{x} 10^{9} / \mathrm{L}$ group. The postoperative intensive care unit (ICU) stay was longer in the preoperative platelet count $<150 \mathrm{x}$ $10^{9} / \mathrm{L}$ group $(16.7 \pm 9.5$ days versus $8.0 \pm 14.5$ days, $p=0.017)$. 
TABLE 1: Clinicopathologic characteristics of patients who underwent extended hepatectomy.

\begin{tabular}{|c|c|}
\hline Variables & $\begin{array}{c}\text { Total } \\
(\mathrm{n}=213)\end{array}$ \\
\hline Age (years) & $60.8 \pm 11.7$ \\
\hline \multicolumn{2}{|l|}{ Gender } \\
\hline Female/male & $108 / 105$ \\
\hline $\operatorname{BMI}\left(\mathrm{kg} / \mathrm{m}^{2}\right)$ & $25.53 \pm 4.44$ \\
\hline \multicolumn{2}{|l|}{ ASA score } \\
\hline Class 1 & $4(2.5 \%)$ \\
\hline Class 2 & $76(46.9 \%)$ \\
\hline Class 3 & $82(50.6 \%)$ \\
\hline \multicolumn{2}{|l|}{ Cirrhosis } \\
\hline Yes & $7(3.2 \%)$ \\
\hline \multicolumn{2}{|c|}{ Indication of extended hepatectomy } \\
\hline Benign liver disease & $9(4.2 \%)$ \\
\hline Primary malignancy & $123(57.7 \%)$ \\
\hline Cholangiocarcinoma & $105(85.4)$ \\
\hline Hepatocellular carcinoma & $18(14.6 \%)$ \\
\hline Metastatic disease & $81(38.0 \%)$ \\
\hline \multicolumn{2}{|l|}{ Preoperative chemotherapy } \\
\hline Yes & $73(35.8 \%)$ \\
\hline \multicolumn{2}{|c|}{ Preoperative platelet count $\left(\times 10^{9} / \mathrm{L}\right)$} \\
\hline Mean (SD) & $300.1 \pm 121.5$ \\
\hline Intraoperative blood loss (ml) & $1638.21 \pm 1535.49$ \\
\hline \multicolumn{2}{|l|}{ Transfusion of RBC } \\
\hline Patient & $60(31.1 \%)$ \\
\hline Unit & $1.52 \pm 3.34$ \\
\hline \multicolumn{2}{|l|}{ Transfusion of FFP } \\
\hline Patient & $44(22.8 \%)$ \\
\hline Unit & $1.43 \pm 3.64$ \\
\hline Operation time (min) & $293.78 \pm 115.15$ \\
\hline PHLF $^{a}$ & $56(26.3 \%)$ \\
\hline Grade A & $16(28.6 \%)$ \\
\hline Grade B & $14(25.0 \%)$ \\
\hline Grade C & $26(46.4 \%)$ \\
\hline Major morbidity ${ }^{\mathrm{b}}$ & $57(26.8 \%)$ \\
\hline ICU stay (days) & $8.14 \pm 13.47$ \\
\hline Hospitalization (days) & $23.43 \pm 16.68$ \\
\hline 30-day mortality & $24(11.3 \%)$ \\
\hline
\end{tabular}

BMI: body mass index; ASA: American Society of Anesthesiologists; SD: standard deviation; RBC: red blood cells; FFP: fresh-frozen plasma; PHLF: posthepatectomy liver failure; ICU: intensive care unit.

${ }^{\text {a }}$ Based on the ISGLS definition.

${ }^{\mathrm{b}}$ Grades III and IV based on the Clavien-Dindo classification.

Furthermore, in the group with platelet count of $<150 \mathrm{x}$ $10^{9} / \mathrm{L}$ PHLF, major complications and 30-day mortality were detected in $58.8 \%, 35.3 \%$, and $35.3 \%$ of patients, respectively (Table 2).

3.1. Predictive Value of Preoperative Platelet Count. To investigate the impact of the preoperative platelet count on postoperative outcomes including PHLF, morbidity, and 30day mortality, we performed univariate and multivariate regression analysis. Univariate analysis (Table 3(a)) revealed that patients with a preoperative platelet count of $<150$ $\mathrm{x} 10^{9} / \mathrm{L}$ are significantly more at risk of PHLF (OR 4.7, 95\% CI 1.7-12.9, $p=0.003$ ). According to univariate analysis, indication of $\mathrm{EH}$, intraoperative blood loss, transfusion of red 
TABLE 2: Clinicopathologic characteristics of patients with a preoperative platelet count of $<150 \times 10^{9} / \mathrm{L}$.

\begin{tabular}{|c|c|}
\hline Variables & $\begin{array}{c}\text { Total } \\
(\mathbf{n}=17)\end{array}$ \\
\hline Age (years) & $63.1 \pm 12.5$ \\
\hline \multicolumn{2}{|l|}{ Gender } \\
\hline Female/male & $8 / 9$ \\
\hline BMI $\left(\mathrm{kg} / \mathrm{m}^{2}\right)$ & $25.08 \pm 3.88$ \\
\hline \multicolumn{2}{|l|}{ ASA score } \\
\hline Class 1 & $0(0.0 \%)$ \\
\hline Class 2 & $8(57.1 \%)$ \\
\hline Class 3 & $6(42.9 \%)$ \\
\hline \multicolumn{2}{|l|}{ Cirrhosis } \\
\hline Yes & $0(0.0 \%)$ \\
\hline \multicolumn{2}{|c|}{ Indication of extended hepatectomy } \\
\hline Benign liver disease & $2(11.8 \%)$ \\
\hline Primary malignancy & $9(52.9 \%)$ \\
\hline Cholangiocarcinoma & $9(100 \%)$ \\
\hline Hepatocellular carcinoma & $0(0.0 \%)$ \\
\hline Metastatic disease & $6(35.3 \%)$ \\
\hline \multicolumn{2}{|l|}{ Preoperative chemotherapy } \\
\hline Yes & $5(29.4 \%)$ \\
\hline \multicolumn{2}{|c|}{ Preoperative platelet count $\left(\times 10^{9} / \mathrm{L}\right)$} \\
\hline Mean (SD) & $122.3 \pm 22.3$ \\
\hline Intraoperative blood loss (ml) & $3352.94 \pm 2019.32$ \\
\hline \multicolumn{2}{|l|}{ Transfusion of RBC } \\
\hline Patient & $8(50.0 \%)$ \\
\hline Unit & $4.38 \pm 6.26$ \\
\hline \multicolumn{2}{|l|}{ Transfusion of FFP } \\
\hline Patient & $9(56.3 \%)$ \\
\hline Unit & $4.31 \pm 5.91$ \\
\hline Operation time (min) & $381.29 \pm 136.05$ \\
\hline PHLF $^{a}$ & $10(58.8 \%)$ \\
\hline Grade A & $0(0.0 \%)$ \\
\hline Grade B & $2(20.0 \%)$ \\
\hline Grade C & $8(80.0 \%)$ \\
\hline Major morbidity ${ }^{b}$ & $6(35.3 \%)$ \\
\hline ICU stay (days) & $16.65 \pm 9.50$ \\
\hline Hospitalization (days) & $30.18 \pm 15.20$ \\
\hline 30-day mortality & $6(35.3 \%)$ \\
\hline
\end{tabular}

BMI: body mass index; ASA: American Society of Anesthesiologists; SD: standard deviation; RBC: red blood cells; FFP: fresh-frozen plasma; PHLF: posthepatectomy liver failure; ICU: intensive care unit.

${ }^{a}$ Based on the ISGLS definition.

${ }^{\mathrm{b}}$ Grades III and IV based on the Clavien-Dindo classification.

blood cells or fresh frozen plasma, and operation time also had a significant impact on PHLF. In contrast, multivariate regression only revealed preoperative thrombocytopenia as an independent preoperative predictor of PHLF (OR 4.4, 95\% CI 1.3-15.0, $p=0.020$ ).
According to multivariate analysis, patient age (OR 1.1, 95\% CI 1.0-1.1, $p=0.001$ ), metastatic liver disease (OR 2.4, 95\% CI 1.1-5.1, $p=0.026$ ), and operation time (OR 1.2, 95\% CI 1.0-1.5, $p=0.042$ ) independently predicted major postoperative morbidities (Table 3(b)). Postoperative major 


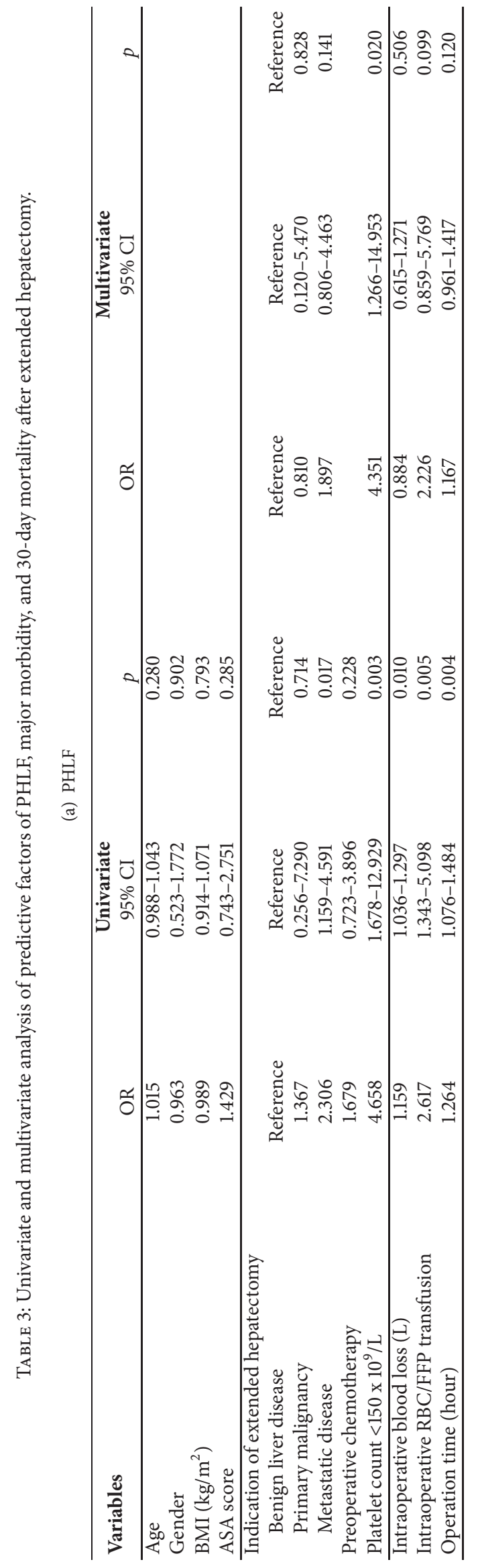




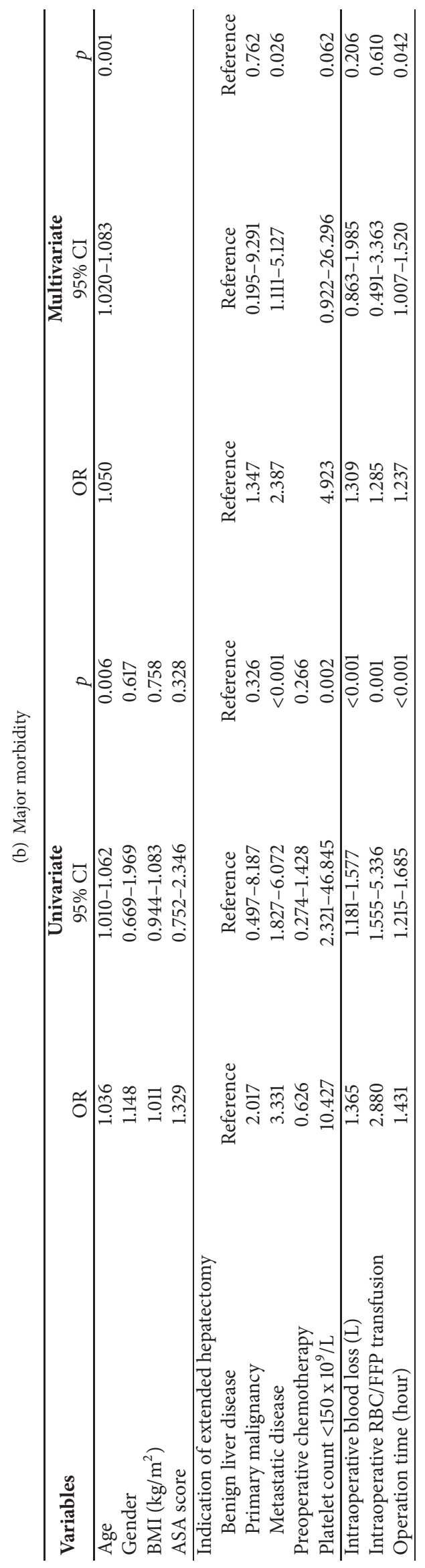




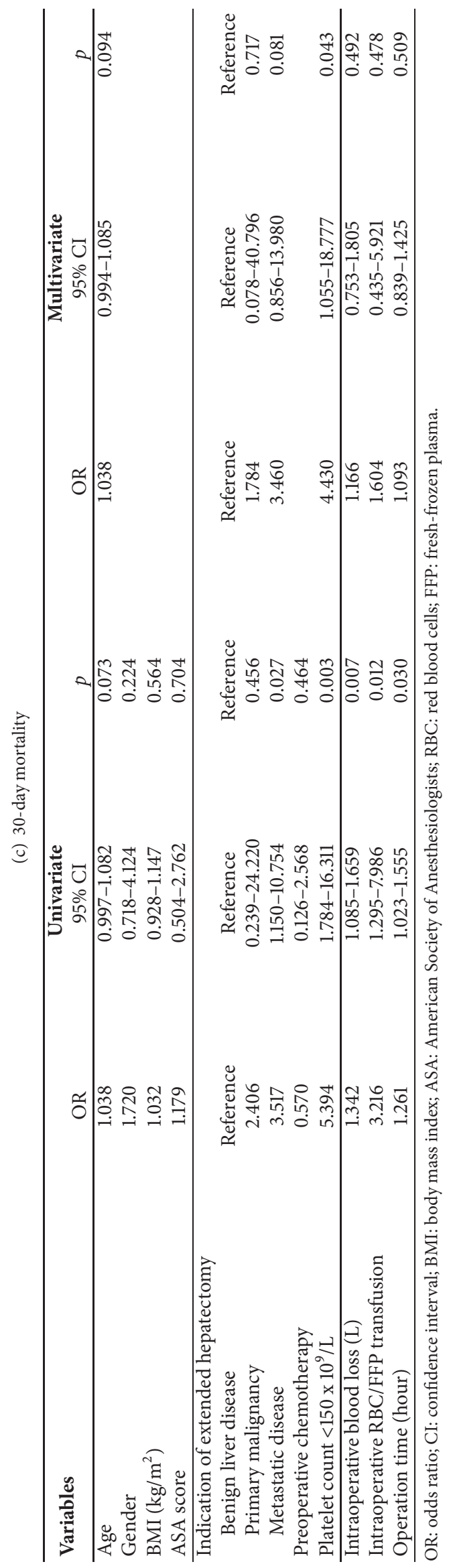




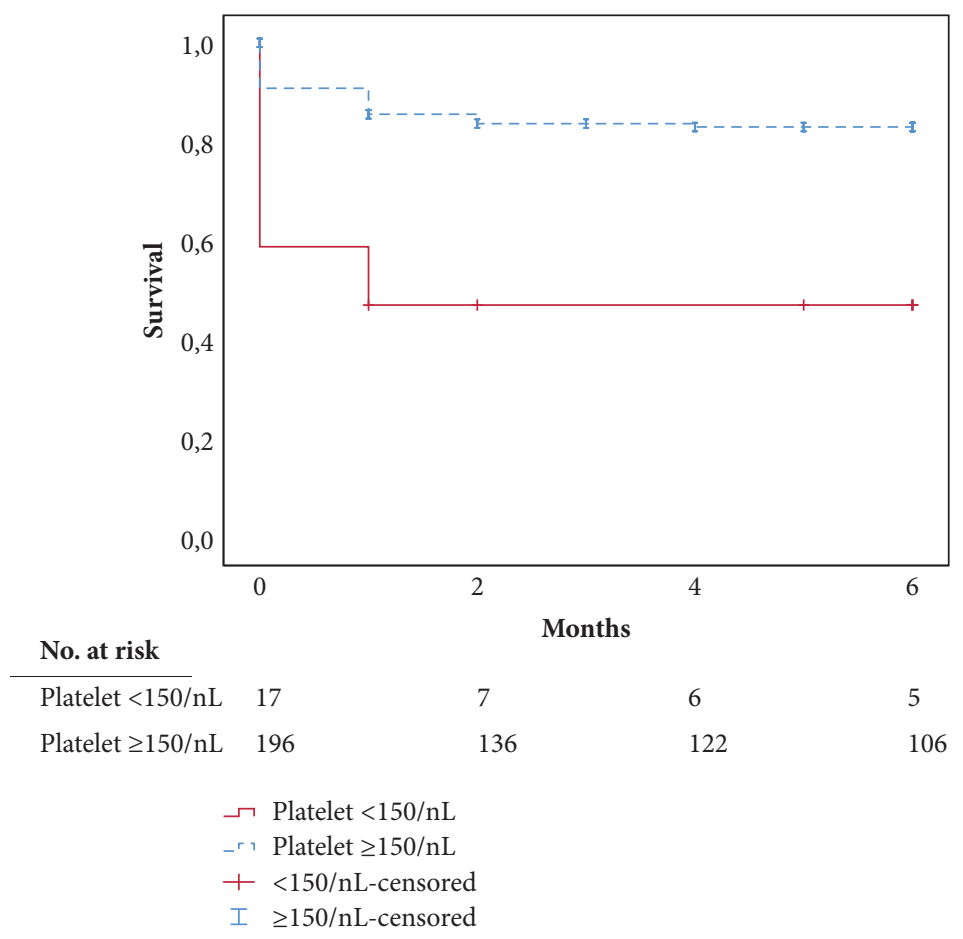

FIGURE 1: Six-month patient survival plot: significantly lower survival rates in patients with low preoperative platelet counts $\left(<150 \mathrm{x} 10^{9} / \mathrm{L}\right)$ compared with normal preoperative platelet counts $(\geq 150)$ (log-rank test $\mathrm{p}<0.001)$.

morbidities were fivefold higher in patients with a preoperative platelet count $<150 \times 10^{9} / \mathrm{L}$, but this difference was not significant (OR 4.9, 95\% CI 0.9-26.3, $p=0.062$ ). Furthermore, multivariate analysis showed that postoperative 30-day mortality was fourfold higher in patients with thrombocytopenia compared with normal platelet counts (Table 3(c)) (OR 4.4, 95\% CI 1.1-18.8, $p=0.043$ ).

After excluding patients with underlying cirrhosis ( $=7$ ), we repeated univariate and multivariate analysis of PHLF, major morbidity and 30-day mortality. As presented in Supplementary Table S1, multivariate analysis demonstrated that PHLF (OR 5.7, 95\% CI 2.6-12.8, $p<0.001$ ) and 30-day mortality (OR 5.9, 95\% CI 1.3-27.1, $p=0.021$ ) were sixfold higher in patients with thrombocytopenia compared with those with normal platelet counts.

3.2. Patient Survival. The six-month survival rate was $80.1 \% \pm 2.8 \%$ in our cohort. Patients with a preoperative platelet count $<150 \times 10^{9} / \mathrm{L}$ had a significantly lower sixmonth survival rate than patients with a preoperative platelet count $\geq 150 \times 10^{9} / \mathrm{L}$ (Figure $1,47.1 \% \pm 12.1 \%$ versus $83.0 \% \pm 2.8 \%$, $\log$-rank $p<0.001)$.

\section{Discussion}

PHLF is a severe and potentially lethal complication after liver resection and is responsible for more than $60 \%$ of mortalities after EH [21, 22]. The high rate of mortality and morbidity following EH is a major concern in field of hepatobiliary surgery. Although low platelet count is associated with increased blood loss and longer operation time, it has been shown that low platelet counts can independently diminish postoperative liver regeneration and increase the risk of PHLF as well mortality $[13,23]$. Recently, the effect of perioperative (preoperative or immediate postoperative) platelet counts on posthepatectomy morbidity and mortality has been investigated [13, 16, 24-28]. However, these studies investigated the predictive role of platelet count in all types of liver resection (minor, major, and extended) and did not distinguish between the different types of resection. PHLF and mortality rates are higher after $\mathrm{EH}$, therefore we believe that the post-EH outcome is more clinically important and that the predictive role of platelet counts should be investigated separately following $\mathrm{EH}$. To do this, we assessed the association of platelet counts and postoperative outcomes in a homogeneous subgroup of liver resection patients who underwent $\mathrm{EH}$.

We demonstrated that a low preoperative platelet count is a predictive factor of PHLF and higher mortality after EH. In our series of EHs, the odds of development of PHLF and 30day mortality in patients with low platelet counts were more than 4 and 6 fold higher than patients with normal platelet count, respectively. Moreover, our results showed that longterm survival was lower in patients with low platelet count than patients with normal platelet count. These findings indicate that a low platelet count independently predicts short- and long-term outcomes after EH. We selected a cutoff value of $150 \times 10^{9} / \mathrm{L}$ for platelet counts because this is the minimum normal platelet count in our center and in most clinical settings.

In agreement with our findings, Alkozai et al. [9] reported a fourfold higher 90-day mortality rate after liver resection in colorectal metastasis patients with thrombocytopenia. They 
also reported delayed postoperative recovery of liver function in patients with low platelet count. However, in their study only $40 \%$ of patients underwent liver resection with remnant liver volume of $<35 \%$. Others have also shown that perioperative thrombocytopenia affects PHLF, morbidity, and mortality rates [24, 28]. Similar to our study, Venkat et al. [15], Maithel et al. [25], and Margonis et al. [26] evaluated the effect of perioperative platelet counts on PHLF and/or mortality with a cut-off value of $150 \times 10^{9} / \mathrm{L}$. Although these studies were not consistent in the platelet cut-off levels and type of liver resection they investigated, each has shown that platelet count $\left(100 \times 10^{9} / \mathrm{L}\right.$ or $\left.150 \times 10^{9} / \mathrm{L}\right)$ is associated with posthepatectomy outcomes. In contrast, some authors have reported no significant association between perioperative platelet count and posthepatectomy outcomes [23, 29]. However, in these studies the platelet count was used as a continuous variable in univariate and/or multivariable analyses. In this regard, it is important to know that the exponentiated coefficient of a continuous predictor in logistic regression is the OR of a oneunit increase in the predictor. A one-unit change in platelet count is not clinically meaningful, which may explain why these authors did not find platelet counts to have significant predictive value.

The predictive role of platelets on PHLF and postoperative mortality may be explained by various mechanisms. One proposed mechanism is the direct promotion of liver regeneration by platelets [30]. This was first suggested by Tomikawa et al. [31] in 1996, who showed that platelets promote liver regeneration after resection by upregulating hepatocyte growth factor. Recent studies have shown that platelets secrete several bioactive factors including serotonin, vascular endothelial growth factor, platelet-derived growth factor, and tumor necrosis factors (TNF- $\alpha$ and TNF- $\beta$ ) to promote liver regeneration in a "direct way" $[23,32,33]$. Another possible mechanism is the "indicative role" of platelets for liver regeneration, which has been described by the parallel regulation of platelet production and liver regeneration by similar factors. Thrombopoietin and interleukin 6 regulate megakaryocyte maturation and platelet production, and can predict the postoperative patient outcome and trigger liver regeneration after hepatectomy $[29,34]$. The risk of intra- and postoperative bleeding is increased in patients with thrombocytopenia. This increased blood loss can lead to additional hypoxic liver damage and therefore impaired hepatocyte function/regeneration. This could also explain PHLF and mortality in patients with thrombocytopenia. Surprisingly, Tomimaru and colleagues [14] showed that the platelet count is a better predictor of PHLF in hepatocellular carcinoma patients than the indocyanine green clearance test.

Furthermore, the association between low preoperative platelet count and high portal vein pressure suggests an alternative mechanism [25]. Low platelet counts in patients with liver disease may be secondary to the increased portal vein pressure and subsequent hypersplenism and increased thrombocyte sequestration in the spleen. Indeed, platelet counts can predict PHLF as an accurate and precise surrogate of the portal vein pressure. However, in our study none of the patients in the thrombocytopenia group had splenomegaly, portal hypertension, or cirrhosis. In addition, we performed a subgroup analysis in noncirrhotic patients to reveal the direct association of platelet count with PHLF and mortality.

The retrospective design is a limitation of the present study. However, platelet counts, other laboratory measurements, morbidity, and mortality of all consecutive patients were all recorded prospectively during the study period. To minimize potential bias and estimate the independent effect of the platelet count as accurately as possible, we controlled factors that are known to affect post-EH morbidity and mortality. These potentially confounding factors included age, gender, BMI, American Society of Anesthesiologists (ASA) score, indication of $\mathrm{EH}$, intraoperative blood loss and transfusion, and operation time using univariate and multivariate regression analyses. Therefore, we evaluated the predictive role of platelet count independent of these factors. Cirrhosis is also associated with low platelet count so may confound the effect of thrombocytopenia on posthepatectomy outcome [35]. Therefore, we performed a subgroup analysis after exclusion of patients with cirrhosis.

\section{Conclusions}

In conclusion, preoperative thrombocytopenia seems to be a reliable predictor of PHLF and increased mortality after EH. This predictive role is independent of other related parameters, including age, cause of hepatectomy, intraoperative blood loss, and duration of surgery. Further randomized studies are required to evaluate the impact of increasing the preoperative platelet count (exogenous platelet infusion versus treatment of the underlying disease) on improving the postoperative outcomes after $\mathrm{EH}$ in patients with thrombocytopenia.

\section{Data Availability}

The data used to support the findings of this study are available from the corresponding author upon request.

\section{Disclosure}

This paper was (1) poster presentation at the annual meeting of the German Association for the Study of the Liver (GASL) on January 27, 2018, in Hamburg, Germany (Golriz, M., O. Ghamarnejad, E. Khajeh et al. Preoperative thrombocytopenia may predict poor surgical outcome after extended hepatectomy. Zeitschrift für Gastroenterologie 56, no. 01 (2018): A4-59.), and (2) poster presentation at the 135th congress of the Deutsche Gesellschaft für Chirurgie (DGCH) on April 18, 2018, in Berlin, Germany (Golriz, M., O. Ghamarnejad, E. Khajeh et al. Preoperative thrombocytopenia may predict poor surgical outcome after extended hepatectomy. Innov Surg Sci 2018; 3, (Suppl 1): s1-s231). This research did not receive any specific grant from funding agencies in the public, commercial, or not-for-profit sectors.

\section{Conflicts of Interest}

The authors declare that there are no conflicts of interest regarding the publication of this article. 


\section{Supplementary Materials}

Supplementary Table S1. Subgroup analysis of predictive factors of PHLF, major morbidity, and 30-day mortality after extended hepatectomy in patients without cirrhosis. (Supplementary Materials)

\section{References}

[1] J.-N. Vauthey, T. M. Pawlik, E. K. Abdalla et al., "Is Extended Hepatectomy for Hepatobiliary Malignancy Justified?" Annals of Surgery, vol. 239, no. 5, pp. 722-732, 2004.

[2] P.-A. Clavien, H. Petrowsky, M. L. DeOliveira, and R. Graf, "Strategies for safer liver surgery and partial liver transplantation," The New England Journal of Medicine, vol. 356, no. 15, pp. 1545-1559, 2007.

[3] M. H. Squires, N. L. Lad, S. B. Fisher et al., "Value of primary operative drain placement after major hepatectomy: A multiinstitutional analysis of 1,041 patients," Journal of the American College of Surgeons, vol. 220, no. 4, pp. 396-402, 2015.

[4] R. T. Poon, S. T. Fan, C. M. Lo et al., "Improving perioperative outcome expands the role of hepatectomy in management of benign and malignant hepatobiliary diseases: Analysis of 1222 consecutive patients from a prospective database," Annals of Surgery, vol. 240, no. 4, pp. 698-710, 2004.

[5] J. Belghiti, K. Hiramatsu, S. Benoist, P. P. Massault, A. Sauvanet, and O. Farges, "Seven hundred forty-seven hepatectomies in the 1990s: an update to evaluate the actual risk of liver resection," Journal of the American College of Surgeons, vol. 191, no. 1, pp. 38-46, 2000.

[6] J. T. Mullen, D. Ribero, S. K. Reddy et al., "Hepatic insufficiency and mortality in 1,059 noncirrhotic patients undergoing major hepatectomy," Journal of the American College of Surgeons, vol. 204, no. 5, pp. 854-862, 2007.

[7] N. N. Rahbari, C. Reissfelder, M. Koch et al., "The predictive value of postoperative clinical risk scores for outcome after hepatic resection: A validation analysis in 807 patients," Annals of Surgical Oncology, vol. 18, no. 13, pp. 3640-3649, 2011.

[8] J. W. Cheng, P. Zhao, J. B. Liu, X. Liu, and X. L. Wu, "Preoperative aspartate aminotransferase-toplatelet ratio index (APRI) is a predictor on postoperative outcomes of hepatocellular carcinoma," Medicine (United States), vol. 95, no. 48, p. e5486, 2016.

[9] S. Balzan, J. Belghiti, O. Farges et al., "The " $50-50$ criteria" on postoperative day 5: an accurate predictor of liver failure and death after hepatectomy," Annals of Surgery, vol. 242, no. 6, pp. 824-829, 2005.

[10] N. Akamatsu, Y. Sugawara, J. Kanako et al., "Low platelet counts and prolonged prothrombin time early after operation predict the 90 days morbidity and mortality in living-donor liver transplantation," Annals of Surgery, vol. 265, no. 1, pp. 166172, 2017.

[11] H. Zou, Y. Wen, K. Yuan, X.-Y. Miao, L. Xiong, and K.-J. Liu, "Combining albumin-bilirubin score with future liver remnant predicts post-hepatectomy liver failure in $\mathrm{HBV}$-associated HCC patients," Liver International, vol. 38, no. 3, pp. 494-502, 2018.

[12] K.-P. Au, S.-C. Chan, K. S.-H. Chok et al., "Child-Pugh Parameters and Platelet Count as an Alternative to ICG Test for Assessing Liver Function for Major Hepatectomy," HPB Surg, vol. 2017, 2017.

[13] E. M. Alkozai, M. W. Nijsten, K. P. De Jong et al., "Immediate postoperative low platelet count is associated with delayed liver function recovery after partial liver resection," Annals of Surgery, vol. 251, no. 2, pp. 300-306, 2010.

[14] Y. Tomimaru, H. Eguchi, K. Gotoh et al., "Platelet count is more useful for predicting posthepatectomy liver failure at surgery for hepatocellular carcinoma than indocyanine green clearance test," Journal of Surgical Oncology, vol. 113, no. 5, pp. 565-569, 2016.

[15] R. Venkat, J. R. Hannallah, R. S. Krouse, and F. B. Maegawa, "Preoperative thrombocytopenia and outcomes of hepatectomy for hepatocellular carcinoma," Journal of Surgical Research, vol. 201, no. 2, pp. 498-505, 2016.

[16] A. Mehrabi, M. Golriz, E. Khajeh et al., "Meta-analysis of the prognostic role of perioperative platelet count in posthepatectomy liver failure and mortality," British Journal of Surgery, vol. 105, no. 10, pp. 1254-1261, 2018.

[17] P. Starlinger, A. Assinger, S. Haegele et al., "Evidence for serotonin as a relevant inducer of liver regeneration after liver resection in humans," Hepatology, vol. 60, no. 1, pp. 257-266, 2014.

[18] J. Belghiti, P.-A. Clavien, E. Gadzijev et al., “The Brisbane 2000 terminology of liver anatomy and resections," $H P B$, vol. 2, no. 3 , pp. 333-339, 2000.

[19] N. N. Rahbari, O. J. Garden, R. Padbury et al., "Posthepatectomy liver failure: a definition and grading by the International Study Group of Liver Surgery (ISGLS)," Surgery, vol. 149, no. 5, pp. 713-724, 2011.

[20] D. Dindo, N. Demartines, and P. Clavien, "Classification of surgical complications: a new proposal with evaluation in a cohort of 6336 patients and results of a survey," Annals of Surgery, vol. 240, no. 2, pp. 205-213, 2004.

[21] M. Donadon, G. Costa, M. Cimino et al., "Safe hepatectomy selection criteria for hepatocellular carcinoma patients:A validation of 336 consecutive hepatectomies. the bilche score," World Journal of Surgery, vol. 39, no. 1, pp. 237-243, 2015.

[22] S. Gruttadauria, A. Tropea, D. Pagano et al., "Mini-Invasive Approach Contributes to Expand the Indication for Liver Resection for Hepatocellular Carcinoma Without Increasing the Incidence of Posthepatectomy Liver Failure and Other Perioperative Complications: A Single-Center Analysis," Journal of Laparoendoscopic \& Advanced Surgical Techniques, vol. 26, no. 6, pp. 439-446, 2016.

[23] J. Cui, E. Heba, C. Hernandez et al., "Magnetic resonance elastography is superior to acoustic radiation force impulse for the Diagnosis of fibrosis in patients with biopsy-proven nonalcoholic fatty liver disease: a prospective study," Hepatology, vol. 63, no. 2, pp. 453-461, 2016.

[24] K. Kaneko, Y. Shirai, T. Wakai, N. Yokoyama, K. Akazawa, and K. Hatakeyama, "Low preoperative platelet counts predict a high mortality after partial hepatectomy in patients with hepatocellular carcinoma," World Journal of Gastroenterology, vol. 11, no. 37, pp. 5888-5892, 2005.

[25] S. K. Maithel, P. J. Kneuertz, and D. A. Kooby, "Importance of low preoperative platelet count in selecting patients for resection of hepatocellular carcinoma: A multi-institutional analysis," Journal of the American College of Surgeons, vol. 212, no. 4, pp. 638-648, 2011.

[26] G. A. Margonis, N. Amini, S. Buettner et al., "Impact of early postoperative platelet count on volumetric liver gain and perioperative outcomes after major liver resection," British Journal of Surgery, vol. 103, no. 7, pp. 899-907, 2016.

[27] C. Riediger, J. Bachmann, A. Hapfelmeier, H. Friess, J. Kleeff, and M. W. Mueller, "Low postoperative platelet count 
is associated with negative outcome after liver resection for hepatocellular carcinoma," Hepato-Gastroenterology, vol. 61, no. 133, pp. 1313-1320, 2014.

[28] H.-Q. Wang, J. Yang, J.-Y. Yang, W.-T. Wang, and L.-N. Yan, "Low immediate postoperative platelet count is associated with hepatic insufficiency after hepatectomy," World Journal of Gastroenterology, vol. 20, no. 33, pp. 11871-11877, 2014.

[29] S. Haegele, F. Offensperger, D. Pereyra et al., "Deficiency in thrombopoietin induction after liver surgery is associated with postoperative liver dysfunction," PLoS ONE, vol. 10, no. 1, 2015.

[30] T. Kurokawa, Y.-W. Zheng, and N. Ohkohchi, "Novel functions of platelets in the liver," Journal of Gastroenterology and Hepatology, vol. 31, no. 4, pp. 745-751, 2016.

[31] M. Tomikawa, M. Hashizume, and H. Highashi, "The role of the spleen, platelets, and plasma hepatocyte growth factor activity on hepatic regeneration in rats," Journal of the American College of Surgeons, vol. 182, no. 1, pp. 12-16, 1996.

[32] B. Aryal, T. Shimizu, J. Kadono et al., "A switch in the dynamics of intra-platelet VEGF-A from cancer to the later phase of liver regeneration after partial hepatectomy in humans," PLoS ONE, vol. 11, no. 3, Article ID e0150446, 2016.

[33] B. Aryal, M. Yamakuchi, and T. Shimizu, "Therapeutic implication of platelets in liver regenerationhopes and hues," Expert Review of Gastroenterology Hepatology, vol. no, 2018.

[34] D. Schmidt-Arras and S. Rose-John, "IL-6 pathway in the liver: from physiopathology to therapy," Journal of Hepatology, vol. 64, no. 6, pp. 1403-1415, 2016.

[35] N. Afdhal, J. McHutchison, R. Brown et al., “Thrombocytopenia associated with chronic liver disease," Journal of Hepatology, vol. 48, no. 6, pp. 1000-1007, 2008. 